\title{
User Interface for a Geo-Temporal Search Service Using DIGMAP Components
}

\author{
Jorge Machado $^{1,2}$, Gilberto Pedrosa ${ }^{1}$, Nuno Freire ${ }^{1}$, Bruno Martins ${ }^{1}$, \\ and Hugo Manguinhas ${ }^{1}$ \\ ${ }^{1}$ INESC-ID / Instituto Superior Técnico \\ ${ }^{2}$ Instituto Politécnico de Portalegre \\ \{jorge.r.machado, gilberto.pedrosa, nuno.freire, \\ bruno.martins, hugo.manguinhas\} @ist.utl.pt
}

\begin{abstract}
This demo presents a user interface for a Geo-Temporal search service built in the sequence of DIGMAP project. DIGMAP was a co-funded European Union project on old digitized maps and deals with resources rich in geographic and temporal information. This search interface followed a mashup approach using existing DIGMAP components: a metadata repository, a text mining tool, a Gazetteer, and a service to generate geographic contextual thumbnails. Google Maps API is used to provide a friendly and interactive user interface. This demo will present the resulting geo-temporal search engine functionalities, whose interface uses WEB 2.0 capabilities to provide contextualization in time and space and text clustering.
\end{abstract}

Keywords: User Interfaces, GeoTemporal Retrieval, Software Architectures, Metadata, Information Retrieval, Lucene.

\section{Introduction}

Nowadays the WEB is used not only by users but also by automatic services using published application programming interfaces (API). This demonstration explores this kind of development, known as mashup, to provide a search engine interface that incorporates geographic, temporal and textual dimensions. The search engine uses the infrastructure of the DIGMAP project ${ }^{1}$. This work follows previous studies presented in [3][4][5] where the authors refer to the relevance of Geo-Temporal user interfaces. Our approach aimed at achieving two goals: provide a friendly and interactive user interface exploring Geo-Temporal properties; separate the geographic and temporal dimensions from the textual one to let the user build the queries in these different dimensions.

To reach first goal are used five internal web services available from DIGMAP were: Nail Map ${ }^{2}$ to generate contextual thumbnails; Metadata Repository ${ }^{3}$ to manage

\footnotetext{
${ }^{1} \mathrm{http} / / /$ www.digmap.eu

${ }^{2} \mathrm{http} / / /$ nail.digmap.eu/

${ }^{3}$ http://repox.ist.utl.pt/
} 
and retrieve metadata records; Gazetteer ${ }^{4}$ together with GeoParser ${ }^{5}$ [1][2] to recognize and disambiguate the names of places and temporal expressions given in the text, also assigning documents to the encompassing geo-temporal scopes that they discuss as a whole. The Google Maps ${ }^{6}$ API's is also used to provide a new interactive search interface. Sections 3 and 4 detail search and results interface respectively.

\section{Search Interface}

The search interface ${ }^{7}$, illustrated in Fig. 1, is structured in four dimensions: text, space, time and collections. In text dimension we provide both a simple form, using only a single text box, and an advanced form combining different metadata elements, are made available. Users can provide restrictions on the textual dimension using one of these forms. The form corresponding to the temporal dimension is presented on top of the form that corresponds to the geographical dimension. In these dimensions we give the user the chance to select a time interval, specified in years, and a geographic box using a map.

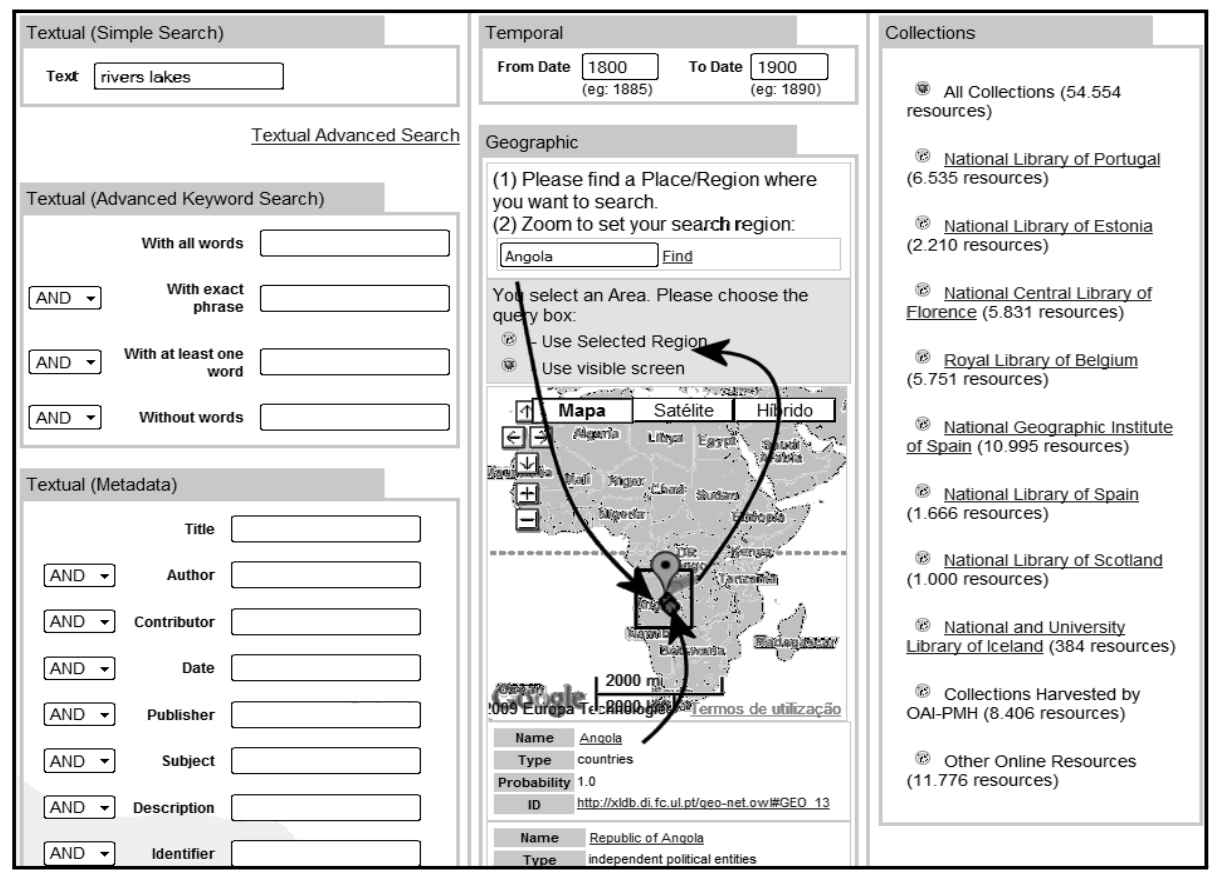

Fig. 1. The DIGMAP Search Service Interface

\footnotetext{
${ }^{4}$ http://gaz.digmap.eu/

${ }^{5} \mathrm{http} / / /$ geoparser.digmap.eu/

${ }^{6} \mathrm{http}: / /$ code.google.com/apis/maps/

${ }^{7}$ http://digmap2.ist.utl.pt:8080/mitra (Experimental Service).
} 
The records were indexed in time, space, text and collections. To index geographic and time dimensions the Geoparser service was used to find geographic scopes and dates, in that sense almost every record, about $90 \%$, have coordinates, latitudes and longitudes, and dates assigned, what turns possible to use this interface with success using a Geo-Temporal retrieval machine. The geographic panel provides a disambiguation service implemented using Geoparser and Gazetteer services from DIGMAP, and Google Maps API to provide an interactive map background. The users could zoom the map to choose the region where they want to search. Some records have geographic polygons attributed automatically by Geoparser or manually by cataloguers. In these cases when the user selects the region marker associated with a polygon the interface ask the user if the search box will be de polygon or the visible region.

\section{Results Interface}

The results page, illustrated in Fig. 2 (in last page), shows the records found for the search requested in Fig. 1. The component Nail Map is invoked in two different moments, first to get one thumbnail with the map miniature, second to get a thumbnail to contextualize the record in the searched region. The time period of the record is also contextualized in the search interval above the context map. When a user clicks on the map or on a record title the interface will invoke the metadata repository to return an HTML version of that record. During the presentation of results the client interface will invoke the search component to obtain clustered text fragments for each one of

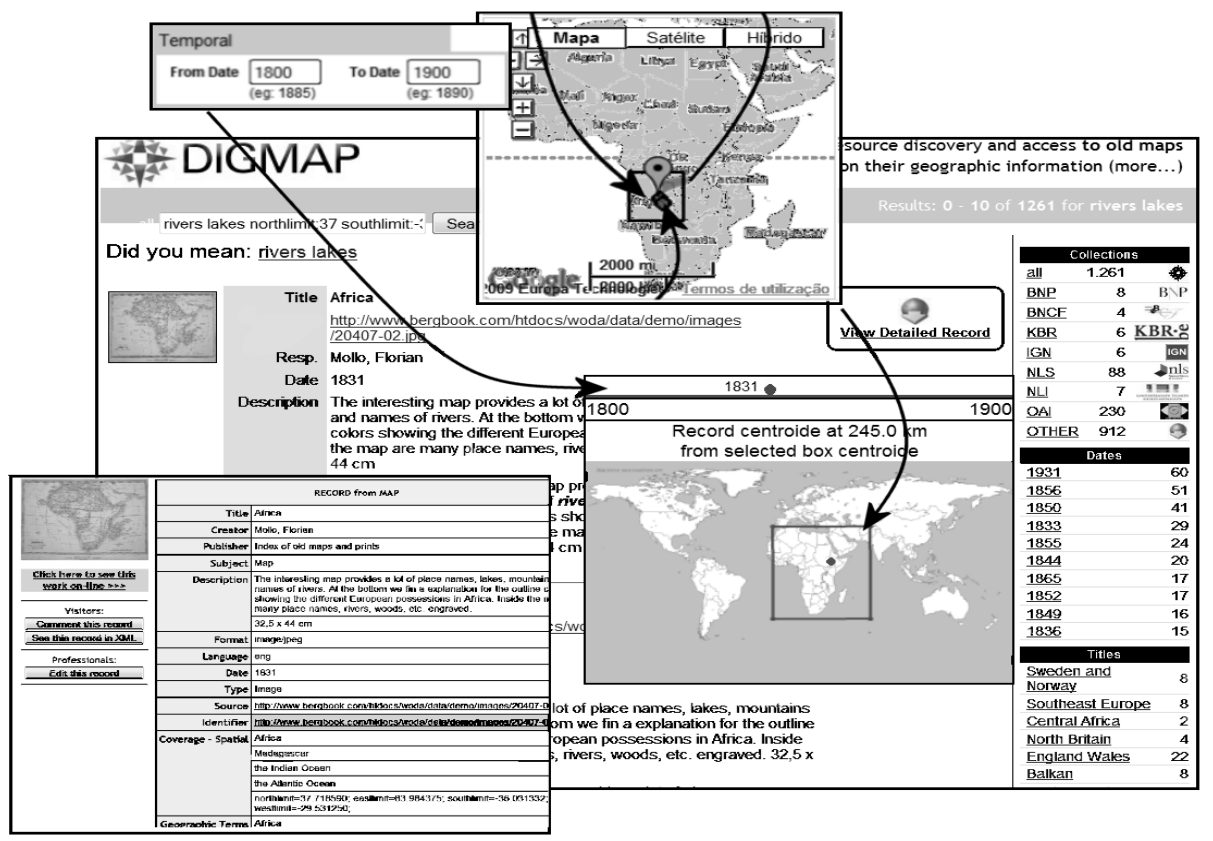

Fig. 2. Search Results in the new DIGMAP search interface 
the metadata fields, dates and collections. All of these calls are transparent to the end user, who just sees as soon as possible a list of results, and after that, a constant evolution of the interface contextualizing him in text, space and time.

\section{Conclusions}

This user interface reveals very good possibilities along a small group of testers whose appreciate the new facilities in Geo-Temporal dimensions. We plan to continue this work adding more features both in Geo-Temporal and Text clustering domain. We plan also to perform usability tests to prove our interface effectiveness join the community.

\section{References}

[1] Manguinhas, H., Martins, B., Borbinha, J.: A Geo-Temporal Web Gazetteer Integrating Data From Multiple Source. In: Proceedings of the 2008 IEEE international Conference on Digital Information Management, November 13-16 (2008)

[2] Martins, B., Freire, N., Borbinha, J.: Complex Data Transformations in Digital Libraries with Spatio-Temporal Information. In: Proceedings of the 2008 International Conference on Asia-Pacific Digital Libraries, December 02-05 (2008)

[3] Buckland, M., Chen, A., Gey, F.C., Larson, R.R., Mostern, R.: Geographic search: catalogs, gazetteers, and maps, College and Research Libraries (2007)

[4] Buckland, M., Chen, A., Gey, F.C., Larson, R.R., Mostern, R., Petras, V.: Geographic Search: Catalogs, Gazetteers, and Maps. College \& Research Libraries 68(5), 376-387 (2007)

[5] Larson, R.R.: Geographic IR and Visualization in Time and Space. In: ACM SIGIR 2008 Thirty-first annual international ACM SIGIR Conference on Research and Development in Information Retrieval, Singapore, July 20-24, 2008, p. 886 (2008) 